\title{
Reprogramming cells to study vacuolar development
}

\author{
Mistianne Feeney ${ }^{1,2,3}{ }^{*}$, Lorenzo Frigerio ${ }^{3}$, Susanne E. Kohalmi ${ }^{1}$, Yuhai Cui ${ }^{1,2}$ and Rima Menassa ${ }^{1,2}$ \\ ${ }^{1}$ Department of Biology, University of Western Ontario, London, ON, Canada \\ ${ }^{2}$ Southern Crop Protection and Food Research Centre, Agriculture and Agri-Food Canada, London, ON, Canada \\ ${ }^{3}$ School of Life Sciences, University of Warwick, Coventry, UK
}

\section{Edited by:}

Marisa Otegui, University of

Wisconsin at Madison, USA

Reviewed by:

Douglas Muench, University of

Calgary, Canada

Diane C. Bassham, lowa State

University, USA

Marcela Rojas-Pierce, North Carolina

State University, USA

*Correspondence:

Mistianne Feeney, School of Life Sciences, University of Warwick,

Coventry CV4 7AL, UK

e-mail:m.feeney@warwick.ac.uk
During vegetative and embryonic developmental transitions, plant cells are massively reorganized to support the activities that will take place during the subsequent developmental phase. Studying cellular and subcellular changes that occur during these short transitional periods can sometimes present challenges, especially when dealing with Arabidopsis thaliana embryo and seed tissues. As a complementary approach, cellular reprogramming can be used as a tool to study these cellular changes in another, more easily accessible, tissue type. To reprogram cells, genetic manipulation of particular regulatory factors that play critical roles in establishing or repressing the seed developmental program can be used to bring about a change of cell fate. During different developmental phases, vacuoles assume different functions and morphologies to respond to the changing needs of the cell. Lytic vacuoles (LVs) and protein storage vacuoles (PSVs) are the two main vacuole types found in flowering plants such as Arabidopsis. Although both are morphologically distinct and carry out unique functions, they also share some similar activities. As the co-existence of the two vacuole types is short-lived in plant cells, how they replace each other has been a long-standing curiosity. To study the LV to PSV transition, LEAFY COTYLEDON2, a key transcriptional regulator of seed development, was overexpressed in vegetative cells to activate the seed developmental program. At the cellular level, Arabidopsis leaf LVs were observed to convert to PSV-like organelles. This presents the opportunity for further research to elucidate the mechanism of LV to PSV transitions. Overall, this example demonstrates the potential usefulness of cellular reprogramming as a method to study cellular processes that occur during developmental transitions.

Keywords: Arabidopsis thaliana, cellular reprogramming, developmental transition, LEAFY COTYLEDON2, lytic vacuole, protein storage vacuole, vacuole biogenesis

\section{INTRODUCTION}

One of the most dramatic cellular changes that occur over the lifetime of a flowering plant happens during the transition between vegetative and embryonic developmental phases. Vegetative cells are massively reorganized to support embryonic development and vice versa (Mansfield and Briarty, 1991, 1992, 1996). The array of organelles present within the cells remains more or less constant, however, vegetative and embryonic cells are morphologically distinct (Figures 1A,B). At the subcellular level, what happens to the organelles during these transitions?

Studying the cellular and subcellular changes that occur during these developmental transitions is challenging. Arabidopsis thaliana is the model organism of the plant community and has been adopted for genetic, physiology, molecular, and developmental biology research (Meyerowitz, 2001; Somerville and Koornneef, 2002). Thus, it is beneficial and practical to relate these areas of study to the cell biology of this plant. In Arabidopsis, however, it is difficult to study the cellular changes that occur during vegetative and embryonic developmental programs on account of the small seed size and technical challenges in isolating embryos from maternal tissues at early stages of embryogenesis (Girke et al., 2000; Xiang et al., 2011; Ibl and Stoger, 2012).
During different developmental stages of the plant life cycle, vacuoles assume diverse functions in response to the changing needs of the cell, and their morphology will be significantly different (Marty, 1999; Zouhar and Rojo, 2009). Due to its sheer size, the lytic vacuole (LV) is the most prominent organelle in the vegetative plant cell. Its counterpart, present in Arabidopsis seeds, is the protein storage vacuole (PSV) which looks nothing like the LV (Figures 1A,B). It is remarkable how such a large organelle (LV) can transform (Zheng and Staehelin, 2011) or be replaced (Hoh etal., 1995) by much smaller and more numerous PSVs and vice versa. Another intriguing issue is how the two vacuoles can have such drastically different functions; the LV is essential for water (Beebo et al., 2009) and ionic homeostasis (Isayenkov et al., 2010), plays a role in shaping vegetative cells (Rojo et al., 2001), and also acts as a cellular waste bin (Li and Vierstra, 2012). In contrast, the PSV stores protein (Scarafoni et al., 2001) and mineral reserves (Otegui etal., 2002) and therefore acts as a cellular pantry. PSVs are present as storage organelles in three of the world's major food crops (Varshney et al., 2012); rice (Kawakatsu et al., 2010), wheat (Regvar et al., 2011), and maize (Reyes etal., 2011) and are prevalent in other important food sources such as nuts, legumes, and other cereals (Bethke et al., 1998; Hara-Nishimura et al., 1998; Robinson et al., 2005). Indeed, 

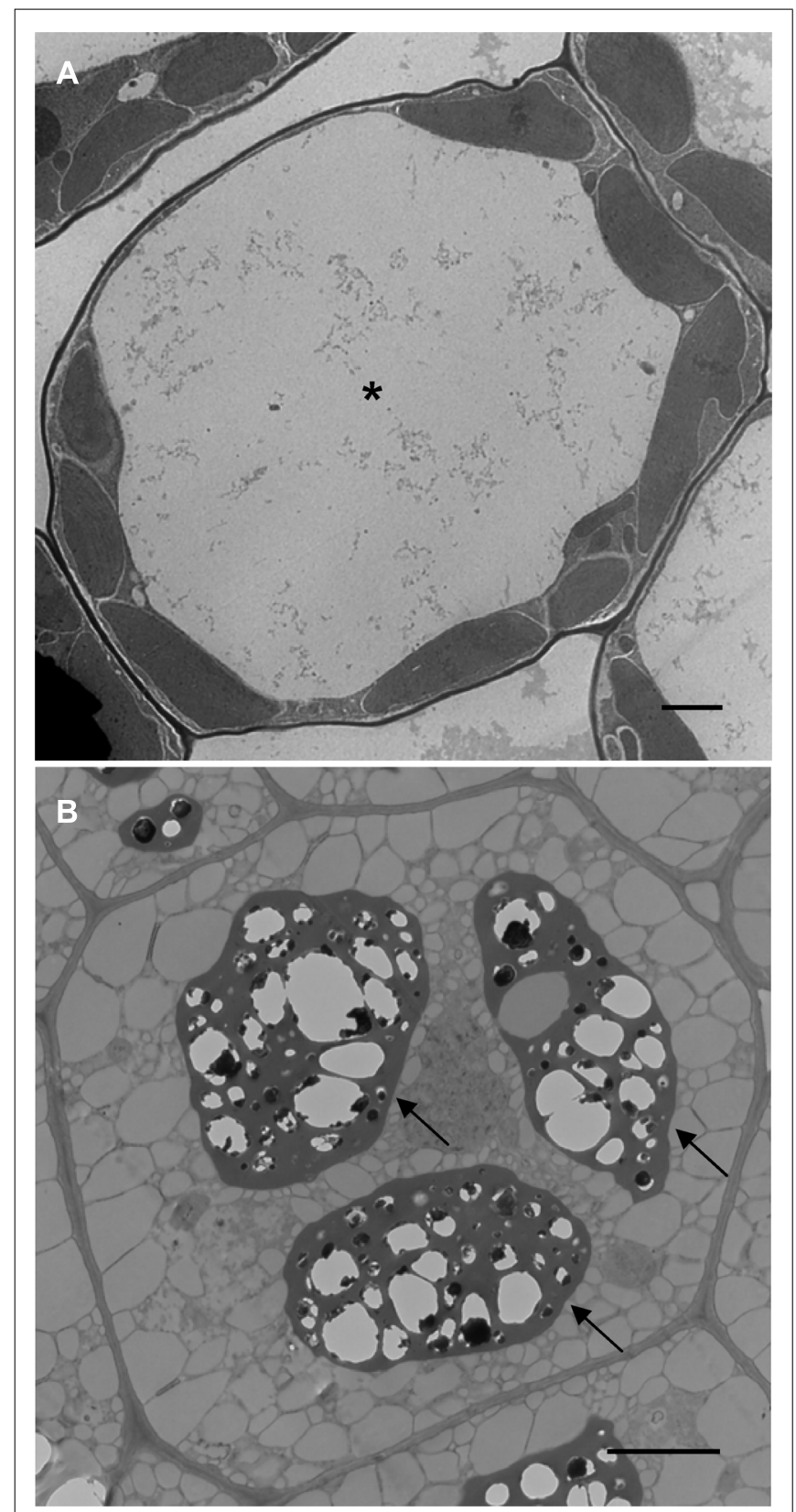

FIGURE 1 | Transmission electron microscope images of Arabidopsis leaf (A) and seed (B) cells. Asterisk shows the LV and arrows point to PSVs. Bars $=2 \mu \mathrm{m}$.

PSVs play an important role in supplying essential nutrients for our dietary needs. Here we discuss how cellular reprogramming can be used to learn more about LV to PSV transitions in Arabidopsis.

\section{LYTIC VACUOLE STRUCTURE AND FUNCTION}

The central LV is the largest and most instantly recognizable organelle in a vegetative plant cell. It can account for up to $90 \%$ of the total cell volume (Jolliffe et al., 2005). As such, the LV squeezes the cytoplasm and other organelles between the tonoplast and plasma membrane (Figure 1A). LVs are present in the cells of young seedlings shortly after germination and generally exist in all cells throughout vegetative growth (Gattolin et al., 2009; Zheng and Staehelin, 2011).

The LV lumen contains water, numerous hydrolytic enzymes, and is maintained at a pH of 5.5-6 (Martinière et al., 2013). The tonoplast plays a major role in maintaining this luminal environment. The tonoplast is a selective membrane that contains a large number of channel and transport proteins that mediate the movement of organic and ionic substances between the cytoplasm and vacuole (Carter et al., 2004; Jaquinod et al., 2007; Müntz, 2007). To maintain an acidic luminal $\mathrm{pH}$, vacuolar ATPase (V-ATPase) and pyrophosphatase (V-PPase) catalyze ATP-dependent proton transfer across the tonoplast. Their activity creates a proton gradient and membrane potential which energizes secondary active transport across the tonoplast (Yang et al., 2007; Krebs et al., 2010). The movement of inorganic metabolites is mediated by specific ion channels (Voelker et al., 2006) or transporters (Brini et al., 2007), while organic substances are moved by ATP-binding cassette (ABC) transporters (Shi et al., 2007; Song et al., 2010). The movement of water across the tonoplast is facilitated by water channels called aquaporins (Maurel et al., 2008). Within the vacuole lumen, numerous hydrolytic enzymes are present such as proteases, glycosidases, lipases, nucleases, and peroxidases (Carter et al., 2004).

The LV participates in diverse physical and metabolic functions that are critical for the survival of a plant. A significant role of the $\mathrm{LV}$ is to allow the cell to increase its size without expending too much energy. This allows lower cost cellular growth as vacuoles largely consist of water and have a low density of organic compounds to synthesize (Zouhar and Rojo, 2009). One universally important function of the LV is its role in maintaining turgor pressure which determines the rigidity of the cell and is important for growth and mechanical stability of the plant (Müntz, 2007). In addition to physical functions, LVs play important metabolic roles by storing a large variety of compounds such as toxins (Riechers et al., 2010), salts (Krebs etal., 2010), heavy metals (Song et al., 2010), pigments (Reuveni et al., 2001; Zhang et al., 2006), and defense compounds (Zhao and Dixon, 2010). The low $\mathrm{pH}$ and numerous hydrolytic enzymes present in the LV lumen allow it to play a fundamental role in the degradation of cytoplasmic materials from small molecules to organelles. This process involves autophagy, a conserved mechanism in eukaryotes whereby cell contents are transferred to the vacuole to be digested and recycled, typically in a non-selective manner (Bassham, 2007; Li and Vierstra, 2012). Generally, a basal level of autophagy functions constitutively for the turnover of cellular components (Wang et al., 2013). However, it can be induced to higher levels during particular developmental stages (Bassham et al., 2006) or in times of cell stress (Liu et al., 2012). Endocytic trafficking of proteins from the plasma membrane is an essential cellular transport system required for cell communication, cellular differentiation, and physiological responses to the environment (Otegui and Spitzer, 2008; Irani and Russinova, 2009; Richter et al., 2009). The LV plays a key role as the cellular endpoint where selected endocytosed proteins are sent for degradation (Otegui and Spitzer, 2008). 


\section{PROTEIN STORAGE VACUOLE STRUCTURE AND FUNCTION}

The PSV is a specialized organelle that is unique to flowering plants (Wang et al., 2012). It is found predominantly in seeds and young meristematic plant cells (Olbrich et al., 2007; Gattolin et al., 2011). In shoot and root meristem cells, the existence of PSVs is thought to be due to the persistence of seed-specific signals in the meristem (Olbrich et al., 2007). Whereas LVs typically occupy most of the cell space, PSVs are much smaller in size and range from 1.5 to $8 \mu \mathrm{m}$ in diameter (Gillespie et al., 2005). PSVs are numerous and are usually positioned close to the center of the cell (Shimada et al., 2008; Figure 1B).

The PSV is a compartmentalized organelle (Jiang et al., 2001; Bolte et al., 2011; Regvar et al., 2011). Comparatively less is known about the PSV tonoplast than the LV tonoplast (Isayenkov et al., 2010) but they are known to share some similarity in their composition of proteins (Hoh et al., 1995; Jiang et al., 2001; Isayenkov et al., 2011). The pH of the PSV lumen varies between 4.9 and 5.5 (Otegui et al., 2006). The defining feature of PSVs is their ability to accumulate seed storage proteins (SSPs). In fact, the appearance of PSVs in embryonic cells coincides with storage reserve accumulation during the maturation phase of embryogenesis (Mansfield and Briarty, 1992). Lesser recognized roles of PSVs are the storage of phosphorus and minerals (Otegui et al., 2002), protective compounds such as lectins (De Hoff et al., 2009) and chitinases (Neuhaus et al., 1991), and proteolytic enzymes (Gruis et al., 2004). Like their LV counterparts, PSVs are also involved in autophagy. PSVs of wheat and maize sequester SSPs from endoplasmic reticulum (ER)-derived protein bodies (Levanony et al., 1992) or prevacuolar-like compartments (Reyes et al., 2011) by autophagic mechanisms, respectively.

\section{VACUOLE CONVERSION DURING EMBRYONIC AND VEGETATIVE GROWTH AND DEVELOPMENT MULTIPLE VACUOLES IN PLANT CELLS}

As discussed, seed plants have two principal, functionally distinct vacuole types, LVs and PSVs (Becker, 2007; Ibl and Stoger, 2012). This has raised questions about whether the two vacuoles co-exist in cells. To address this question, the two vacuole types must be distinguished. LVs and PSVs can be differentiated by the presence of tonoplast intrinsic protein (TIP) isoforms (Jauh et al., 1999; Gattolin etal., 2010). TIPs are aquaporins that are specifically localized to tonoplasts (Maurel et al., 2009). Arabidopsis has 10 TIP isoforms and two of these are used to discriminate between LVs and PSVs, TIP1;1 and TIP3;1, respectively (Johanson et al., 2001; Gattolin et al., 2010). In addition, a small number of soluble proteins have been shown to reside exclusively within the lumen of each vacuole type and thus also serve as markers to discriminate between both vacuole types. For example, the cysteine protease aleurain is targeted to the LV (Ahmed et al., 2000), and phaseolin and $2 \mathrm{~S}$ albumin, the major SSPs of common bean and Arabidopsis, respectively, are targeted to PSVs (Frigerio et al., 1998; Miao et al., 2008). LVs and PSVs were originally proposed to co-exist in plant cells based on TIP and soluble protein localization studies undertaken in the 1990s (Paris et al., 1996; Park et al., 2004). This idea was attractive because it provided a convenient explanation for the diverse sorting signals and routes that proteins follow to arrive at the two vacuole types (Vitale and Raikhel, 1999;
Vitale and Hinz, 2005; Frigerio et al., 2008; Rojo and Denecke, 2008). This theory was subsequently challenged as more insight was gained by studying TIP expression patterns. TIP3;1 and TIP1;1 expression were discovered to be tissue- and development-specific rather than organelle-specific (Hunter et al., 2007; Olbrich et al., 2007). Recently, a detailed map of TIP expression patterns in Arabidopsis has been produced to better understand the distribution pattern of these vacuolar markers (Gattolin et al., 2009, 2010, 2011). However, some differences in TIP expression patterns have been reported (Bolte et al., 2011). The current general view is that most cells contain one vacuolar type. However, both vacuoles have been shown to co-exist in some cells but their co-existence is usually short-lived (Hoh et al., 1995; Frigerio et al., 2008; Zheng and Staehelin, 2011).

\section{BIOGENESIS OF VACUOLES DURING DEVELOPMENTAL TRANSITIONS LV to PSV transition}

During Arabidopsis embryogenesis, a large LV forms in the fertilized zygote. The zygote then divides to produce a vacuolated basal cell (which will form the suspensor) and a non-vacuolated apical cell (which will give rise to the embryo; De Smet et al., 2010). As the embryo continues to divide, LVs develop in all cells (Zouhar and Rojo, 2009). During the maturation phase of embryogenesis, LVs are replaced by PSVs which will accumulate SSPs and mineral reserves (Mansfield and Briarty, 1991).

\section{PSV to LV transition}

During germination, storage reserves are mobilized to provide nutrients and energy for the growing embryo (Pritchard et al., 2002; Tan-Wilson and Wilson, 2012). As protein and mineral reserves are released, PSVs are replaced by LVs. The LV subsequently increases in volume to create turgor and support cell growth and expansion (Mansfield and Briarty, 1996). In Arabidopsis, it takes approximately $3.5 \mathrm{~d}$ for the PSV to LV transition to occur (Hunter et al., 2007). PSV to LV transitions are also observed in root cells. PSVs are present in the radicle as it emerges from the seed coat. As the root elongates, meristematic regions of the root tip retain PSVs while PSVs transition to LVs in the distal regions of elongating roots (Olbrich et al., 2007; Gattolin et al., 2011; Zheng and Staehelin, 2011).

How do such morphologically distinct vacuoles replace each other in the cell during vegetative and embryonic transitions? We envisage two possible scenarios: either a new vacuolar type arises by remodeling of the pre-existing vacuole, or a new vacuole is formed de novo and very rapidly supersedes the existing one.

\section{Vacuole remodeling}

One means by which different vacuole types may replace each other is by remodeling or reprogramming the vacuole that is already present in the cell. Several studies support this hypothesis. During Arabidopsis germination and seedling development, Mansfield and Briarty (1996) observed multiple PSVs fusing to form a LV after the mobilization of most protein reserves. Olbrich et al. (2007) observed the formation of a single vacuole in barley and pea root tip cells. Close to the root tip, cells contain PSVs with TIP3;1 in their tonoplast. As root cells differentiate, the enlarging vacuole becomes a PSV-LV hybrid as indicated by the presence 
of both TIP3;1 and TIP1;1 and storage proteins in the lumen. The hybrid vacuole then gradually differentiates into a LV with increasing amounts of TIP1;1 and decreasing amounts of TIP3;1 in the tonoplast. Similarly, Zheng and Staehelin (2011) observed that PSVs in tobacco root tips were transformed into LVs. This PSV to LV transition involves unique, highly tissue-specific spatial and temporal changes in vacuole architecture. In addition, within some cell types, the transformation was shown to involve autophagosome formation and engulfment by the developing LV (Zheng and Staehelin, 2011).

\section{De novo vacuole formation}

An alternative hypothesis to explain how different vacuole types arise involves the independent generation of a vacuole within a cell that already has a pre-existing vacuole. A key study to support this theory was conducted by Hoh et al. (1995) who investigated the formation of PSVs in pea cotyledons during seed development. The authors observed the development of a tubular PSV structure which overtook the pre-existing LV. A second example backing this hypothesis is the demonstration that vacuoles can be regenerated from evacuolated protoplasts (Hörtensteiner et al., 1992; Di Sansebastiano et al., 2001). Using autophagy inhibitors, an autophagy-like mechanism was demonstrated to be involved in vacuole biogenesis of evacuolated tobacco protoplasts (Yano et al., 2007). However, the mechanism is distinct from conserved autophagy pathways (Bassham, 2007). Most recently, Viotti et al. (2013) also demonstrated the formation of autophagosome-like structures which give rise to LVs in Arabidopsis root tip cells but showed that these structures were not formed by the core autophagy machinery.

If the de novo theory holds true, then where does the membrane for a new vacuole originate? If an autophagy-like mechanism is involved in biogenesis, perhaps the tonoplast is generated by a process similar to autophagosome formation ( $\mathrm{Li}$ and Vierstra, 2012). In autophagosome formation, the first step is the formation of an isolation membrane which occurs in the cytoplasm close to the vacuole. This process involves the recruitment of several autophagy-related (ATG) proteins which assemble in a coordinated manner to form a cup-shaped membrane structure that elongates and eventually engulfs material to be transported to the vacuole (Mizushima, 2007; Nakatogawa et al., 2009). Mutants defective in vacuole formation have been identified (Zouhar and Rojo, 2009). Most mutations affect factors involved in membrane fusion. An essential gene involved in vacuole biogenesis has been identified as VACUOLELESS1 (VCL1) through a mutant screen (Rojo et al., 2001). Loss-of-function $v c l 1$ embryos were unable to form vacuoles. Mutants accumulated large numbers of autophagosomes which were unable to fuse to form the vacuole but instead would fuse with the plasma membrane and deliver their vacuolar contents to the apoplasm. Thus, VCL1 is proposed to be involved in regulating the fusion of autophagosomes to form a LV (Zouhar and Rojo, 2009). Tonoplasts have also been proposed to originate from the ER or the Golgi apparatus (Marty, 1978; Robinson and Hinz, 1997; Staehelin, 1997; Neuhaus and Rogers, 1998). Using mutants and pharmacological inhibitors which affect the biosynthetic secretory pathway, Viotti etal. (2013) demonstrated that tonoplast proteins and lipids were derived from the ER and were delivered directly, via a Golgi-independent route, to form the LV tonoplast.

\section{REPROGRAMMING CELLS TO STUDY ORGANELLE DYNAMICS: VACUOLES}

As an alternative to studying PSV formation in developing seeds, we asked if it was possible to induce the formation of PSVs in vegetative cells. Cues prompting vegetative cells to switch to PSV formation are not well understood. Despite the fact that SSPs are the major storage reserves that accumulate in PSVs, their forced synthesis in vegetative tissues has not been demonstrated to promote PSV formation. Constitutive expression of phaseolin in alfalfa vegetative tissues did not result in a significant accumulation of the protein in cells of non-seed organs (Bagga et al., 1992). Further, phaseolin was shown to accumulate and be degraded in the LV and, in part, secreted upon overexpression in tobacco (Frigerio et al., 1998). In transgenic Arabidopsis plants overexpressing a chimeric $2 S$ albumin gene, novel precursor-accumulating (PAC)-like vesicles were induced to form in leaves (Hayashi et al., 1999). Within a plant, PSVs are abundant in seed tissues and are also observed in meristematic cells in vegetative tissues (Olbrich et al., 2007). Thus it seems that for PSVs to exist, cells must be programmed to be in an embryonic state.

Genetic research has uncovered a number of genes that play critical roles in establishing or repressing embryonic cell fate (Braybrook and Harada, 2008; Zhang and Ogas, 2009; Jia etal., 2013b). Several transcription factors such as $L E A F Y$ COTYLEDON1 (LEC1; Lotan et al., 1998), LEC2 (Stone et al., 2001), FUSCA3 (FUS3; Gazzarrini etal., 2004), BABYBOOM (BBM; Boutilier etal., 2002), WUSCHEL (WUS; Zuo etal., 2002), EMBRYOMAKER (EMK; Tsuwamoto et al., 2010), and MYB118 (Wang et al., 2009) act during embryogenesis to promote seed developmental programs. In contrast, negative seed regulators such as PICKLE (PKL; Ogas et al., 1999), POLYCOMB REPRESSIVE COMPLEX 2 (PRC2; Bouyer et al., 2011), SET DOMAIN GROUP 8 (SDG8; Tang et al., 2012b), BRAHMA (BRM; Tang et al., 2008), VP1/ABSCISIC ACID INSENSITIVE 3-LIKE (VAL) genes (Suzuki et al., 2007) and microRNA166 (miR166; Tang et al., 2012a) are responsible for suppressing the seed program in vegetative tissues. Thus, overexpression or downregulation of these positive and negative seed regulators, respectively, will induce a seed-specific developmental program causing vegetative tissues to exhibit embryonic characteristics. This scenario presents an opportunity to study the cellular and subcellular changes that take place during this developmental transition.

The maturation phase of embryogenesis represents an exciting window of time to study organelle dynamics. One of the most distinctive activities that take place is a high level of storage reserve accumulation (Mansfield and Briarty, 1992). In Arabidopsis seeds, lipids and proteins usually accumulate up to $30-40 \%$ each of the seed dry weight. Lipids are stored in oil bodies which originate from the ER membrane (Hsieh and Huang, 2004) and PSVs arise to accumulate SSPs as discussed (Mansfield and Briarty, 1992). The events that take place during the maturation phase are controlled by the complex seed regulatory network introduced above (Santos-Mendoza et al., 2008). A key part of this control is achieved 
through the activities of a small number of transcriptional regulators; LEC1, LEC2, FUS3, and ABSCISIC ACID INSENSITIVE3 ( $A B I 3$; Zhang and Ogas, 2009). Genetic studies showed that vegetative tissues overexpressing these transcription factors would begin to exhibit seed traits (Gazzarrini et al., 2004; Kagaya et al., 2005; Stone et al., 2008; Junker et al., 2012).

A wealth of genetic knowledge has been gathered on LEC2 activities (Stone etal., 2001, 2008; Kroj et al., 2003; Santos Mendoza et al., 2005; Braybrook et al., 2006; To et al., 2006; Baud et al., 2007). To learn more about the cellular changes that occur during the vegetative to embryonic transition, a dexamethasone (DEX)-inducible LEC2-GR expression system was exploited in Arabidopsis (Feeney et al., 2013). The overexpression of LEC2 triggers massive cellular reorganization in leaves and causes these vegetative organs to exhibit embryonic characteristics. Among the many cellular and subcellular changes, the replacement of LVs with PSV-like organelles was most notable. In these leaf cells, the large $\mathrm{LV}$ is replaced by smaller and more numerous vacuoles that contain SSP aggregates. Upon further investigation using immunogold labeling with tonoplast and luminal markers, it was established that the small vacuoles had the features of developing PSVs (Feeney et al., 2013). Indeed, the presence of the PSV-specific TIP3;1 protein on the tonoplast (Gattolin et al., 2011) and accumulation of SSPs within the lumen of the small vacuoles indicates that the leaf vacuoles assumed a storage role (Jauh et al., 1999; Hunter et al.,
2007; Olbrich et al., 2007). Furthermore, confocal analysis revealed a unique embryo-like vacuolar morphology (Figures 2B,E). To visualize the tonoplast of these developing leaf PSVs, 35S:LEC2GR plants co-expressing TIP3;1-YFP under its native promoter was generated. The native TIP3;1 promoter is developmentally regulated and thus the TIP3;1-YFP fusion is specifically expressed in seed tissues and accumulated on PSV tonoplasts (Hunter et al., 2007). As DEX-induced plants overexpressing LEC2 began to acquire embryogenic characteristics (Feeney et al., 2013), TIP3;1YFP became detectable on the tonoplast of leaf cells, indicating that vacuoles were PSVs (Figures 2B,E). Highly fluorescent TIP3;1YFP-labeled tonoplast folds and bulbs appeared (Saito et al., 2002, 2011). These are characteristic vacuolar morphologies of young cells (Figure 2A). However, the tonoplast also retained the characteristic configuration of a large LV lining the periphery of the cell. To highlight the vacuole lumen, tissues were stained with neutral red and revealed that the lumen appears to occupy the entire leaf cell (Figure 2B) unlike seed PSVs (Figure 2C). In addition, vacuolar lumina began to exhibit autofluorescence (Figure 2E), which was not observed in uninduced leaves (Figure 2D). Autofluorescent vacuole lumina are usually observed in seed PSVs (Figure 2F; Fuji et al., 2007; Hunter et al., 2007; Bolte et al., 2011). Therefore, leaf vacuole tonoplasts were extensively remodeled but their lumina remained large and filled the entire cell (Figures 2B,E). These results suggest that in response to DEX-inducible LEC2

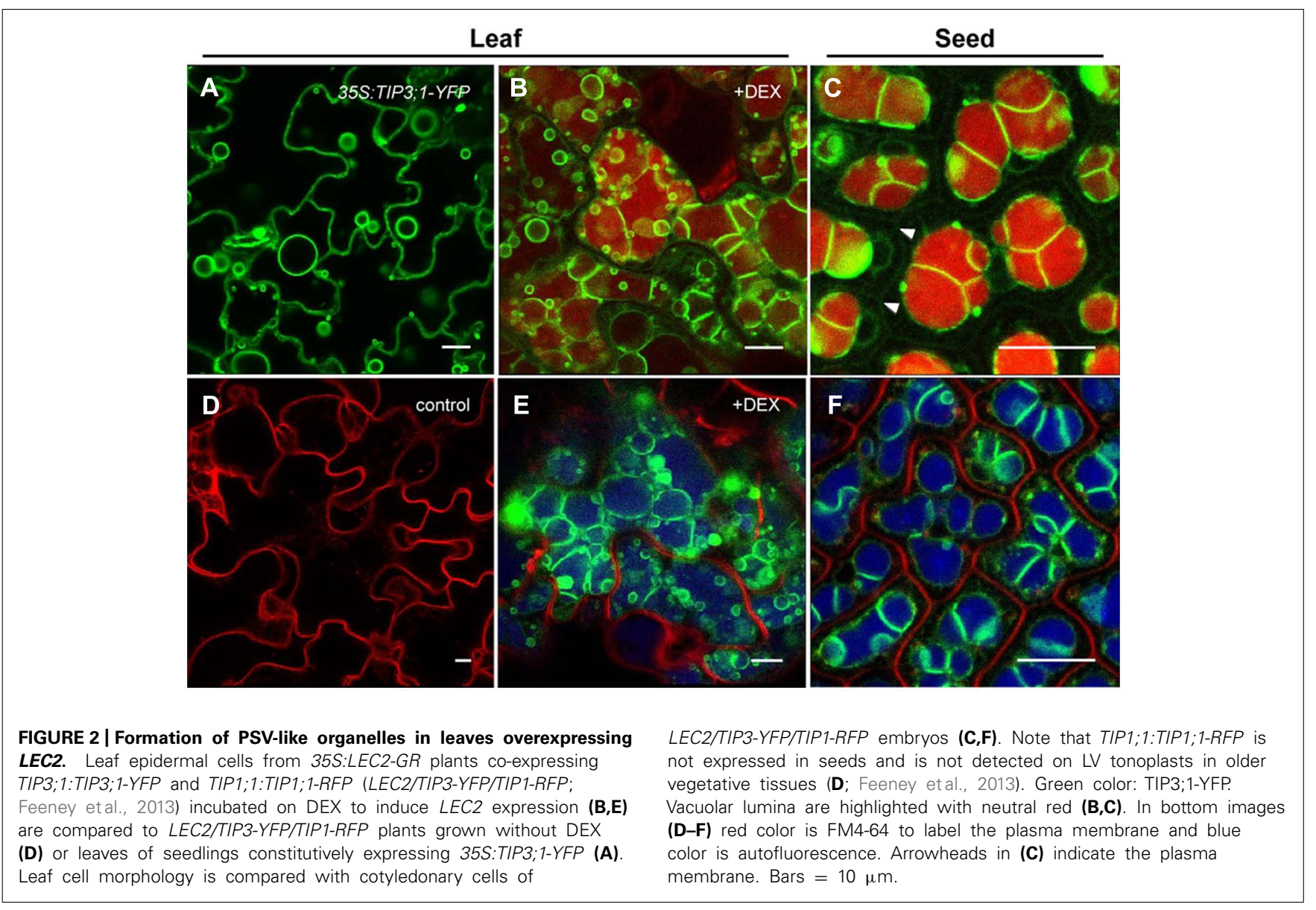


overexpression, leaf LVs are replaced by PSV-like organelles that bear a resemblance to both LVs and PSVs. Therefore, it appears that as the Arabidopsis leaf LV is replaced by a PSV, the LV tonoplast remodels before being replaced by smaller-sized PSVs (Feeney etal., 2013). While these observations seem to point toward a remodeling of existing LVs into PSVs, they do not rule out the possibility of de novo biogenesis of PSVs, which may overlap with $\mathrm{LV}$ remodeling. The next question that will need to be addressed is therefore how exactly PSVs replace LVs during LEC2-induced leaf cell reprogramming.

\section{A CHALLENGE OF CELLULAR REPROGRAMMING: KNOW YOUR GENETIC REGULATOR}

A number of genetic factors have been revealed to promote cellular reprogramming and some are highlighted above. To bring about cellular changes, many of these factors are involved in complex genetic, biochemical, and physiological interactions (Gazzarrini et al., 2004; Gutierrez et al., 2007; Le et al., 2010; Xiang et al., 2011). These factors may act in a hierarchical order (Kagaya et al., 2005; To et al., 2006) and may become active at slightly different developmental times (Le et al., 2010; Willmann et al., 2011). Thus, while many of the factors display a redundancy in promoting cellular reprogramming, they may also display unique activities that affect distinct cellular processes (Baud et al., 2007; Jia et al., 2013a). These aspects should be taken into consideration when choosing a reprogramming system to study a particular cellular process. In the case of vacuoles, several factors may cause vacuolar transitions in vegetative tissues. We have demonstrated that $L E C 2$ overexpression causes LVs to transition to PSVs and results from overexpression studies with LEC1 (Junker et al., 2012) and FUS3 (Gazzarrini et al., 2004) are suggestive that these transcription factors can also bring about a change in vacuole type.

\section{CONCLUSION}

Cellular reprogramming may be a useful means of allowing the study of cellular processes that take place during the short transitional period between two developmental programs. Several genes have been discovered that control embryonic cell identity by establishing or repressing the seed developmental program (Braybrook and Harada, 2008; Zhang and Ogas, 2009; Jia et al., 2013b). In the example presented in this review, overexpression of $L E C 2$ was used to activate the seed developmental program in Arabidopsis leaves (Santos Mendoza et al., 2005; Stone et al., 2008). This system could then be used to study the cellular and subcellular changes that ensue during the vegetative to embryonic transition (Feeney et al., 2013). The observation that LVs were replaced by PSV-like organelles in leaves presents an opportunity to elucidate the mechanism of LV to PSV transitions in Arabidopsis. Overall, we foresee that the major advantage of cellular reprogramming in vegetative tissues is to provide a convenient and complementary system in which to study cellular processes that normally occur during developmental transitions in developing seeds-tissues that are technically challenging to work with.

\section{ACKNOWLEDGMENTS}

We thank J. J. Harada for his ideas and helpful discussions and J. McNeil for reviewing the manuscript. This work was supported by the A-base Funding Program of Agriculture and Agri-Food Canada. Work in the Frigerio lab was funded by a grant from the Leverhulme Trust (RPG-327).

\section{REFERENCES}

Ahmed, S. U., Rojo, E., Kovaleva, V., Venkataraman, S., Dombrowski, J. E., Matsuoka, K., et al. (2000). The plant vacuolar sorting receptor AtELP is involved in transport of NH2-terminal propeptide-containing vacuolar proteins in Arabidopsis thaliana. J. Cell Biol. 149, 1335-1344. doi: 10.1083/jcb.149.7.1335

Bagga, S., Sutton, D., Kemp, J., and Sengupta-Gopalan, C. (1992). Constitutive expression of the $\beta$-phaseolin gene in different tissues of transgenic alfalfa does not ensure phaseolin accumulation in non-seed tissue. Plant Mol. Biol. 19, 951-958. doi: 10.1007/BF00040527

Bassham, D. C., Laporte, M., Marty, F., Moriyasu, Y., Ohsumi, Y., Olsen, L. J., et al. (2006). Autophagy in development and stress responses of plants. Autophagy 2, 2-11.

Bassham, D. C. (2007). Plant autophagy - more than a starvation response. Curr. Opin. Plant Biol. 10, 587-593. doi: 10.1016/j.pbi.2007.06.006

Baud, S., Santos Mendoza, M., To, A., Harscoët, E., Lepiniec, L., and Dubreucq, B. (2007). WRINKLED1 specifies the regulatory action of LEAFY COTYLEDON2 towards fatty acid metabolism during seed maturation in Arabidopsis. Plant J. 50, 825-838. doi: 10.1111/j.1365-313X.2007.03092.x

Becker, B. (2007). Function and evolution of the vacuolar compartment in green algae and land plants (Viridiplantae). Int. Rev. Cytol. 264, 1-24. doi: 10.1016/S0074-7696(07)64001-7

Beebo, A., Thomas, D., Der, C., Sanchez, L., Leborgne-Castel, N., Marty, F., et al. (2009). Life with and without AtTIP1;1, an Arabidopsis aquaporin preferentially localized in the apposing tonoplasts of adjacent vacuoles. Plant Mol. Biol. 70, 193-209. doi: 10.1007/s11103-009-9465-2

Bethke, P. C., Swanson, S. J., Hillmer, S., and Jones, R. L. (1998). From storage compartment to lytic organelle: the metamorphosis of the aleurone protein storage vacuole. Ann. Bot. 82, 399-412. doi: 10.1006/anbo.1998.0702

Bolte, S., Lanquar, V., Soler, M.-N., Beebo, A., Satiat-Jeunemaître, B., Bouhidel, K., et al. (2011). Distinct lytic vacuolar compartments are embedded inside the protein storage vacuole of dry and germinating Arabidopsis thaliana seeds. Plant Cell Physiol. 52, 1142-1152. doi: 10.1093/pcp/pcr065

Boutilier, K., Offringa, R., Sharma, V. K., Kieft, H., Ouellet, T., Zhang, L., et al. (2002). Ectopic expression of BABY BOOM triggers a conversion from vegetative to embryonic growth. Plant Cell 14, 1737-1749. doi: 10.1105/tpc.001941

Bouyer, D., Roudier, F., Heese, M., Andersen, E. D., Gey, D., Nowack, M. K., et al. (2011). Polycomb repressive complex 2 controls the embryo-to-seedling phase transition. PLoS Genet. 7:e1002014. doi: 10.1371/journal.pgen.1002014

Braybrook, S. A., and Harada, J. J. (2008). LECs go crazy in embryo development. Trends Plant Sci. 13, 624-630. doi: 10.1016/j.tplants.2008.09.008

Braybrook, S. A., Stone, S. L., Park, S., Bui, A. Q., Le, B. H., Fischer, R. L., et al. (2006). Genes directly regulated by LEAFY COTYLEDON2 provide insight into the control of embryo maturation and somatic embryogenesis. Proc. Natl. Acad. Sci. U.S.A. 103, 3468-3473. doi: 10.1073/pnas.0511331103

Brini, F., Hanin, M., Mezghani, I., Berkowitz, G. A., and Masmoudi, K. (2007). Overexpression of wheat $\mathrm{Na}+/ \mathrm{H}+$ antiporter TNHX1 and $\mathrm{H}+$-pyrophosphatase TVP1 improve salt- and drought-stress tolerance in Arabidopsis thaliana plants. J. Exp. Bot. 58, 301-308. doi: 10.1093/jxb/erl251

Carter, C., Pan, S., Zouhar, J., Avila, E. L., Girke, T., and Raikhel, N. V. (2004). The vegetative vacuole proteome of Arabidopsis thaliana reveals predicted and unexpected proteins. Plant Cell 16, 3285-3303. doi: 10.1105/tpc.104.027078

De Hoff, P., Brill, L., and Hirsch, A. (2009). Plant lectins: the ties that bind in root symbiosis and plant defense. Mol. Genet. Genomics 282, 1-15. doi: 10.1007/s00438-009-0460-8

De Smet, I., Lau, S., Mayer, U., and Jürgens, G. (2010). Embryogenesis - the humble beginnings of plant life. Plant J. 61, 959-970. doi: 10.1111/j.1365313X.2010.04143. $\mathrm{x}$

Di Sansebastiano, G. P., Paris, N., Marc-Martin, S., and Neuhaus, J.-M. (2001). Regeneration of a lytic central vacuole and of neutral peripheral vacuoles can be visualized by green fluorescent proteins targeted to either type of vacuoles. Plant Physiol. 126, 78-86. doi: 10.1104/pp.126.1.78

Feeney, M., Frigerio, L., Cui, Y., and Menassa, R. (2013). Following vegetative to embryonic cellular changes in leaves of Arabidopsis overexpressing LEAFY COTYLEDON2. Plant Physiol. 162, 1881-1896. doi: 10.1104/pp.113.220996 
Frigerio, L., De Virgilio, M., Prada, A., Faoro, F., and Vitale, A. (1998). Sorting of phaseolin to the vacuole is saturable and requires a short $\mathrm{C}$-terminal peptide. Plant Cell 10, 1031-1042.

Frigerio, L., Hinz, G., and Robinson, D. G. (2008). Multiple vacuoles in plant cells: rule or exception? Traffic 9, 1564-1570. doi: 10.1111/j.1600-0854.2008.00776.x

Fuji, K., Shimada, T., Takahashi, H., Tamura, K., Koumoto, Y., Utsumi, S., et al. (2007). Arabidopsis vacuolar sorting mutants (green fluorescent seed) can be identified efficiently by secretion of vacuole-targeted green fluorescent protein in their seeds. Plant Cell 19, 597-609. doi: 10.1105/tpc.106.045997

Gattolin, S., Sorieul, M., and Frigerio, L. (2010). Tonoplast intrinsic proteins and vacuolar identity. Biochem. Soc. Trans. 38, 769-773. doi: 10.1042/BST0380769

Gattolin, S., Sorieul, M., and Frigerio, L. (2011). Mapping of tonoplast intrinsic proteins in maturing and germinating Arabidopsis seeds reveals dual localization of embryonic TIPs to the tonoplast and plasma membrane. Mol. Plant 4, 180-189. doi: $10.1093 / \mathrm{mp} / \mathrm{ssq} 051$

Gattolin, S., Sorieul, M., Hunter, P., Khonsari, R., and Frigerio, L. (2009). In vivo imaging of the tonoplast intrinsic protein family in Arabidopsis roots. BMC Plant Biol. 9:133. doi: 10.1186/1471-2229-9-133

Gazzarrini, S., Tsuchiya, Y., Lumba, S., Okamoto, M., and McCourt, P. (2004). The transcription factor FUSCA3 controls developmental timing in Arabidopsis through the hormones gibberellin and abscisic acid. Dev. Cell 7, 373-385. doi: 10.1016/j.devcel.2004.06.017

Gillespie, J., Rogers, S. W., Deery, M., Dupree, P., and Rogers, J. C. (2005). A unique family of proteins associated with internalized membranes in protein storage vacuoles of the Brassicaceae. Plant J. 41, 429-441. doi: 10.1111/j.1365313X.2004.02303.x

Girke, T., Todd, J., Ruuska, S., White, J., Benning, C., and Ohlrogge, J. (2000). Microarray analysis of developing Arabidopsis seeds. Plant Physiol. 124, 15701581. doi: 10.1104/pp.124.4.1570

Gruis, D., Schulze, J., and Jung, R. (2004). Storage protein accumulation in the absence of the vacuolar processing enzyme family of cysteine proteases. Plant Cell 16, 270-290. doi: 10.1105/tpc.016378

Gutierrez, L., Van Wuytswinkel, O., Castelain, M., and Bellini, C. (2007). Combined networks regulating seed maturation. Trends Plant Sci. 12, 294-300. doi: 10.1016/j.tplants.2007.06.003

Hara-Nishimura, I., Shimada, T., Hatano, K., Takeuchi, Y., and Nishimura, M. (1998). Transport of storage proteins to protein storage vacuoles is mediated by large precursor-accumulating vesicles. Plant Cell 10, 825-836. doi:10.1105/tpc.10.5.825

Hayashi, M., Toriyama, K., Kondo, M., Hara-Nishimura, I., and Nishimura, M. (1999). Accumulation of a fusion protein containing $2 S$ albumin induces novel vesicles in vegetative cells of Arabidopsis. Plant Cell Physiol. 40, 263-272. doi: 10.1093/oxfordjournals.pcp.a029537

Hoh, B., Hinz, G., Jeong, B. K., and Robinson, D. G. (1995). Protein storage vacuoles form de novo during pea cotyledon development. J. Cell Sci. 108, 299-310.

Hörtensteiner, S., Martinoia, E., and Amrhein, N. (1992). Reappearance of hydrolytic activities and tonoplast proteins in the regenerated vacuole of evacuolated protoplasts. Planta 187, 113-121. doi: 10.1007/BF00201632

Hsieh, K., and Huang, A. H. C. (2004). Endoplasmic reticulum, oleosins, and oils in seeds and tapetum cells. Plant Physiol. 136, 3427-3434. doi: 10.1104/pp.104.051060

Hunter, P. R., Craddock, C. P., Di Benedetto, S., Roberts, L. M., and Frigerio, L. (2007). Fluorescent reporter proteins for the tonoplast and the vacuolar lumen identify a single vacuolar compartment in Arabidopsis cells. Plant Physiol. 145, 1371-1382. doi: 10.1104/pp.107.103945

Ibl, V., and Stoger, E. (2012). The formation, function and fate of protein storage compartments in seeds. Protoplasma 249, 379-392. doi: 10.1007/s00709-0110288-Z

Irani, N. G., and Russinova, E. (2009). Receptor endocytosis and signaling in plants. Curr. Opin. Plant Biol. 12, 653-659. doi: 10.1016/j.pbi.2009.09.011

Isayenkov, S., Isner, J. C., and Maathuis, F. J. M. (2010). Vacuolar ion channels: roles in plant nutrition and signalling. FEBS Lett. 584, 1982-1988. doi: 10.1016/j.febslet.2010.02.050

Isayenkov, S., Isner, J.-C., and Maathuis, F. J. M. (2011). Rice two-pore K+ channels are expressed in different types of vacuoles. Plant Cell 23, 756-768. doi: $10.1105 /$ tpc. 110.081463

Jaquinod, M., Villiers, F., Kieffer-Jaquinod, S., Hugouvieux, V., Bruley, C., Garin, J., et al. (2007). A proteomics dissection of Arabidopsis thaliana vacuoles isolated from cell culture. Mol. Cell. Proteomics 6, 394-412. doi: 10.1074/mcp.M600250MCP200

Jauh, G.-Y., Phillips, T. E., and Rogers, J. C. (1999). Tonoplast intrinsic protein isoforms as markers for vacuolar functions. Plant Cell 11, 1867-1882. doi: 10.1105/tpc.11.10.1867

Jia, H., McCarty, D. R., and Suzuki, M. (2013a). Distinct roles of LAFL network genes in promoting the embryonic seedling fate in the absence of VAL repression. Plant Physiol. 163, 1293-1305. doi: 10.1104/pp.113.220988

Jia, H., Suzuki, M., and McCarty, D. R. (2013b). Regulation of the seed to seedling developmental phase transition by the LAFL and VAL transcription factor networks. Wiley Interdiscip. Rev. Dev. Biol. doi: 10.1002/wdev.126

Jiang, L., Phillips, T. E., Hamm, C. A., Drozdowicz, Y. M., Rea, P. A., Maeshima, M., et al. (2001). The protein storage vacuole: a unique compound organelle. J. Cell Biol. 155, 991-1002. doi: 10.1083/jcb.200107012

Johanson, U., Karlsson, M., Johansson, I., Gustavsson, S., Sjövall, S., Fraysse, L., et al. (2001). The complete set of genes encoding major intrinsic proteins in Arabidopsis provides a framework for a new nomenclature for major intrinsic proteins in plants. Plant Physiol. 126, 1358-1369. doi: 10.1104/pp.126.4.1358

Jolliffe, N. A., Craddock, C. P., and Frigerio, L. (2005). Pathways for protein transport to seed storage vacuoles. Biochem. Soc. Trans. 33, 1016-1018. doi: 10.1042/BST20051016

Junker, A., Mönke, G., Rutten, T., Keilwagen, J., Seifert, M., Thi, T. M. N., et al. (2012). Elongation-related functions of LEAFY COTYLEDON1 during the development of Arabidopsis thaliana. Plant J. 71, 427-442. doi: 10.1111/j.1365-313X.2012.04999.X

Kagaya, Y., Toyoshima, R., Okuda, R., Usui, H., Yamamoto, A., and Hattori, T. (2005). LEAFY COTYLEDON1 controls seed storage protein genes through its regulation of FUSCA3 and ABSCISIC ACID INSENSITIVE3. Plant Cell Physiol. 46, 399-406. doi: 10.1093/pcp/pci048

Kawakatsu, T., Hirose, S., Yasuda, H., and Takaiwa, F. (2010). Reducing rice seed storage protein accumulation leads to changes in nutrient quality and storage organelle formation. Plant Physiol. 154, 1842-1854. doi: 10.1104/pp.110.164343

Krebs, M., Beyhl, D., Görlich, E., Al-Rasheid, K. A. S., Marten, I., Stierhof, Y.-D., et al. (2010). Arabidopsis V-ATPase activity at the tonoplast is required for efficient nutrient storage but not for sodium accumulation. Proc. Natl. Acad. Sci. U.S.A. 107, 3251-3256. doi: 10.1073/pnas.0913035107

Kroj, T., Savino, G., Valon, C., Giraudat, J., and Parcy, F. (2003). Regulation of storage protein gene expression in Arabidopsis. Development 130, 6065-6073. doi: 10.1242/dev.00814

Le, B. H., Cheng, C., Bui, A. Q., Wagmaister, J. A., Henry, K. F., Pelletier, J., et al. (2010). Global analysis of gene activity during Arabidopsis seed development and identification of seed-specific transcription factors. Proc. Natl. Acad. Sci. U.S.A. 107, 8063-8070. doi: 10.1073/pnas. 1003530107

Levanony, H., Rubin, R., Altschuler, Y., and Galili, G. (1992). Evidence for a novel route of wheat storage proteins to vacuoles. J. Cell Biol. 119, 1117-1128. doi: 10.1083/jcb.119.5.1117

Li, F., and Vierstra, R. D. (2012). Autophagy: a multifaceted intracellular system for bulk and selective recycling. Trends Plant Sci. 17, 526-537. doi: 10.1016/j.tplants.2012.05.006

Liu, Y., Burgos, J. S., Deng, Y., Srivastava, R., Howell, S. H., and Bassham, D. C. (2012). Degradation of the endoplasmic reticulum by autophagy during endoplasmic reticulum stress in Arabidopsis. Plant Cell 24, 4635-4651. doi: 10.1105/tpc.112.101535

Lotan, T., Ohto, M.-a., Yee, K. M., West, M. A. L., Lo, R., Kwong, R. W., et al. (1998). Arabidopsis LEAFY COTYLEDON1 is sufficient to induce embryo development in vegetative cells. Cell 93, 1195-1205. doi: 10.1016/S0092-8674(00)81463-4

Mansfield, S. G., and Briarty, L. G. (1991). Early embryogenesis in Arabidopsis thaliana. II. The developing embryo. Can. J. Bot. 69, 461-476. doi: 10.1139/b91063

Mansfield, S. G., and Briarty, L. G. (1992). Cotyledon cell development in Arabidopsis thaliana during reserve deposition. Can. J. Bot. 70, 151-164. doi: 10.1139/b92-021 Mansfield, S. G., and Briarty, L. G. (1996). The dynamics of seedling and cotyledon cell development in Arabidopsis thaliana during reserve mobilization. Int. J. Plant Sci. 157, 280-295. doi: 10.1086/297347

Martinière, A., Bassil, E., Jublanc, E., Alcon, C., Reguera, M., Sentenac, H., et al. (2013). In vivo intracellular $\mathrm{pH}$ measurements in tobacco and Arabidopsis reveal an unexpected $\mathrm{pH}$ gradient in the endomembrane system. Plant Cell doi: 10.1105/tpc.113.116897 [Epub ahead of print]. 
Marty, F. (1978). Cytochemical studies on GERL, provacuoles, and vacuoles in root meristematic cells of Euphorbia. Proc. Natl. Acad. Sci. U.S.A. 75, 852-856. doi: 10.1073/pnas.75.2.852

Marty, F. (1999). Plant vacuoles. Plant Cell 11, 587-599. doi: 10.1105/tpc.11.4.587

Maurel, C., Santoni, V., Luu, D.-T., Wudick, M. M., and Verdoucq, L. (2009). The cellular dynamics of plant aquaporin expression and functions. Curr. Opin. Plant Biol. 12, 690-698. doi: 10.1016/j.pbi.2009.09.002

Maurel, C., Verdoucq, L., Luu, D.-T., and Santoni, V. (2008). Plant aquaporins: membrane channels with multiple integrated functions. Annu. Rev. Plant Biol. 59, 595-624. doi: 10.1146/annurev.arplant.59.032607.092734

Meyerowitz, E. M. (2001). Prehistory and history of Arabidopsis research. Plant Physiol. 125, 15-19. doi: 10.1104/pp.125.1.15

Miao, Y., Li, K. Y., Li, H.-Y., Yao, X., and Jiang, L. (2008). The vacuolar transport of aleurain-GFP and $2 S$ albumin-GFP fusions is mediated by the same pre-vacuolar compartments in tobacco BY-2 and Arabidopsis suspension cultured cells. Plant J. 56, 824-839. doi: 10.1111/j.1365-313X.2008.03645.x

Mizushima, N. (2007). Autophagy: process and function. Genes Dev. 21, 2861-2873. doi: 10.1101/gad.1599207

Müntz, K. (2007). Protein dynamics and proteolysis in plant vacuoles. J. Exp. Biol. 58, 2391-2407. doi: 10.1093/jxb/erm089

Nakatogawa, H., Suzuki, K., Kamada, Y., and Ohsumi, Y. (2009). Dynamics and diversity in autophagy mechanisms: lessons from yeast. Nat. Rev. Mol. Cell Biol. 10, 458-467. doi: 10.1038/nrm 2708

Neuhaus, J.-M., and Rogers, J. C. (1998). Sorting of proteins to vacuoles in plant cells. Plant Mol. Biol. 38, 127-144. doi: 10.1023/A:1006032627036

Neuhaus, J.-M., Sticher, L., Meins, F., and Boller, T. (1991). A short $\mathrm{C}$-terminal sequence is necessary and sufficient for the targeting of chitinases to the plant vacuole. Proc. Natl. Acad. Sci. U.S.A. 88, 10362-10366. doi: 10.1073/pnas.88.22.10362

Ogas, J., Kaufmann, S., Henderson, J., and Somerville, C. (1999). PICKLE is a CHD3 chromatin-remodeling factor that regulates the transition from embryonic to vegetative development in Arabidopsis. Proc. Natl. Acad. Sci. U.S.A. 96, 1383913844. doi: 10.1073/pnas.96.24.13839

Olbrich, A., Hillmer, S., Hinz, G., Oliviusson, P., and Robinson, D. G. (2007). Newly formed vacuoles in root meristems of barley and pea seedlings have characteristics of both protein storage and lytic vacuoles. Plant Physiol. 145, 1383-1394. doi: 10.1104/pp.107.108985

Otegui, M. S., Capp, R., and Staehelin, L. A. (2002). Developing seeds of Arabidopsis store different minerals in two types of vacuoles and in the endoplasmic reticulum. Plant Cell 14, 1311-1327. doi: 10.1105/tpc.010486

Otegui, M. S., Herder, R., Schulze, J., Jung, R., and Staehelin, L. A. (2006). The proteolytic processing of seed storage proteins in Arabidopsis embryo cells starts in the multivesicular bodies. Plant Cell 18, 2567-2581. doi: 10.1105/tpc.106.040931

Otegui, M. S., and Spitzer, C. (2008). Endosomal functions in plants. Traffic 9, 1589-1598. doi: 10.1111/j.1600-0854.2008.00787.x

Paris, N., Stanley, C. M., Jones, R. L., and Rogers, J. C. (1996). Plant cells contain two functionally distinct vacuolar compartments. Cell 85, 563-572. doi: 10.1016/S0092-8674(00)81256-8

Park, M., Kim, S. J., Vitale, A., and Hwang, I. (2004). Identification of the protein storage vacuole and protein targeting to the vacuole in leaf cells of three plant species. Plant Physiol. 134, 625-639. doi: 10.1104/pp.103.030635

Pritchard, S. L., Charlton, W. L., Baker, A., and Graham, I. A. (2002). Germination and storage reserve mobilization are regulated independently in Arabidopsis. Plant J. 31, 639-647. doi: 10.1046/j.1365-313X.2002.01376.x

Regvar, M., Eichert, D., Kaulich, B., Gianoncelli, A., Pongrac, P., Vogel-Mikuš, K., et al. (2011). New insights into globoids of protein storage vacuoles in wheat aleurone using synchrotron soft X-ray microscopy. J. Exp. Bot. 62, 3929-3939. doi: 10.1093/jxb/err090

Reuveni, M., Evenor, D., Artzi, B., Perl, A., and Erner, Y. (2001). Decrease in vacuolar $\mathrm{pH}$ during petunia flower opening is reflected in the activity of tonoplast H+-ATPase. J. Plant Physiol. 158, 991-998. doi: 10.1078/0176-161700302

Reyes, F. C., Chung, T., Holding, D., Jung, R., Vierstra, R., and Otegui, M. S. (2011). Delivery of prolamins to the protein storage vacuole in maize aleurone cells. Plant Cell 23, 769-784. doi: 10.1105/tpc.110.082156

Richter, S., Voß, U., and Jürgens, G. (2009). Post-Golgi traffic in plants. Traffic 10, 819-828. doi: 10.1111/j.1600-0854.2009. 00916.x
Riechers, D. E., Kreuz, K., and Zhang, Q. (2010). Detoxification without intoxication: herbicide safeners activate plant defense gene expression. Plant Physiol. 153, 3-13. doi: 10.1104/pp.110.153601

Robinson, D. G., and Hinz, G. (1997). Vacuole biogenesis and protein transport to the plant vacuole: a comparison with the yeast vacuole and the mammalian lysosome. Protoplasma 197, 1-25. doi: 10.1007/BF01279880

Robinson, D. G., Oliviusson, P., and Hinz, G. (2005). Protein sorting to the storage vacuoles of plants: a critical appraisal. Traffic 6, 615-625. doi: 10.1111/j.16000854.2005.00303.x

Rojo, E., and Denecke, J. (2008). What is moving in the secretory pathway of plants? Plant Physiol. 147, 1493-1503. doi: 10.1104/pp.108.124552

Rojo, E., Gillmor, C. S., Kovaleva, V., Somerville, C. R., and Raikhel, N. V. (2001). VACUOLELESS 1 is an essential gene required for vacuole formation and morphogenesis in Arabidopsis. Dev. Cell 1, 303-310. doi: 10.1016/S1534-5807(01)00024-7

Saito, C., Ueda, T., Abe, H., Wada, Y., Kuroiwa, T., Hisada, A., et al. (2002). A complex and mobile structure forms a distinct subregion within the continuous vacuolar membrane in young cotyledons of Arabidopsis. Plant J. 29, 245-255. doi: 10.1046/j.0960-7412.2001.01189.x

Saito, C., Uemura, T., Awai, C., Tominaga, M., Ebine, K., Ito, J., et al. (2011). The occurrence of 'bulbs', a complex configuration of the vacuolar membrane, is affected by mutations of vacuolar SNARE and phospholipase in Arabidopsis. Plant J. 68, 64-73. doi: 10.1111/j.1365-313X.2011.04665.x

Santos-Mendoza, M., Dubreucq, B., Baud, S., Parcy, F., Caboche, M., and Lepiniec, L. (2008). Deciphering gene regulatory networks that control seed development and maturation in Arabidopsis. Plant J. 54, 608-620. doi: 10.1111/j.1365313X.2008.03461.x

Santos Mendoza, M., Dubreucq, B., Miquel, M., Caboche, M., and Lepiniec, L. (2005). LEAFY COTYLEDON2 activation is sufficient to trigger the accumulation of oil and seed specific mRNAs in Arabidopsis leaves. FEBS Lett. 579, 4666-4670. doi: 10.1016/j.febslet.2005.07.037

Scarafoni, A., Carzaniga, R., Harris, N., and Croy, R. D. (2001). Manipulation of the napin primary structure alters its packaging and deposition in transgenic tobacco (Nicotiana tabacum L.) seeds. Plant Mol. Biol. 46, 727-739. doi: 10.1023/A:1011675918805

Shi, J., Wang, H., Schellin, K., Li, B., Faller, M., Stoop, J. M., et al. (2007). Embryospecific silencing of a transporter reduces phytic acid content of maize and soybean seeds. Nat. Biotechnol. 25, 930-937. doi: 10.1038/nbt1322

Shimada, T. L., Shimada, T., Takahashi, H., Fukao, Y., and Hara-Nishimura, I. (2008). A novel role for oleosins in freezing tolerance of oilseeds in Arabidopsis thaliana. Plant J. 55, 798-809. doi: 10.1111/j.1365-313X.2008.03553.x

Somerville, C., and Koornneef, M. (2002). A fortunate choice: the history of Arabidopsis as a model plant. Nat. Rev. Genet. 3, 883-889. doi: 10.1038/nrg927

Song, W.-Y., Park, J., Mendoza-Cózatl, D. G., Suter-Grotemeyer, M., Shim, D. Hörtensteiner, S., et al. (2010). Arsenic tolerance in Arabidopsis is mediated by two ABCC-type phytochelatin transporters. Proc. Natl. Acad. Sci. U.S.A. 107, 21187-21192. doi: 10.1073/pnas.1013964107

Staehelin, L. A. (1997). The plant ER: a dynamic organelle composed of a large number of discrete functional domains. Plant J. 11, 1151-1165. doi: 10.1046/j.1365-313X.1997.11061151.x

Stone, S. L., Braybrook, S. A., Paula, S. L., Kwong, L. W., Meuser, J., Pelletier, J., et al. (2008). Arabidopsis LEAFY COTYLEDON2 induces maturation traits and auxin activity: implications for somatic embryogenesis. Proc. Natl. Acad. Sci. U.S.A. 105, 3151-3156. doi: 10.1073/pnas.0712364105

Stone, S. L., Kwong, L. W., Yee, K. M., Pelletier, J., Lepiniec, L., Fischer, R. L., et al. (2001). LEAFY COTYLEDON2 encodes a B3 domain transcription factor that induces embryo development. Proc. Natl. Acad. Sci. U.S.A. 98, 11806-11811. doi: 10.1073/pnas.201413498

Suzuki, M., Wang, H. H. Y., and McCarty, D. R. (2007). Repression of the LEAFY COTYLEDON 1/B3 regulatory network in plant embryo development by VP1/ABSCISIC ACID INSENSITIVE 3-LIKE B3 genes. Plant Physiol. 143, 902-911. doi: 10.1104/pp.106.092320

Tang, X., Bian, S., Tang, M., Lu, Q., Li, S., Liu, X., et al. (2012a). MicroRNA-mediated repression of the seed maturation program during vegetative development in Arabidopsis. PLoS Genet. 8:e1003091. doi: 10.1371/journal.pgen.1003091

Tang, X., Lim, M.-H., Pelletier, J., Tang, M., Nguyen, V., Keller, W. A., et al. (2012b). Synergistic repression of the embryonic programme by SET DOMAIN GROUP 8 and EMBRYONIC FLOWER 2 in Arabidopsis seedlings. J. Exp. Bot. 63, 1391-1404 doi: $10.1093 /$ jxb/err383 
Tang, X., Hou, A., Babu, M., Nguyen, V., Hurtado, L., Lu, Q., et al. (2008). The Arabidopsis BRAHMA chromatin-remodeling ATPase is involved in repression of seed maturation genes in leaves. Plant Physiol. 147, 1143-1157. doi: 10.1104/pp.108.121996

Tan-Wilson, A. L., and Wilson, K. A. (2012). Mobilization of seed protein reserves. Physiol. Plant. 145, 140-153. doi: 10.1111/j.1399-3054.2011.01535.x

To, A., Valon, C., Savino, G., Guilleminot, J., Devic, M., Giraudat, J., et al. (2006). A network of local and redundant gene regulation governs Arabidopsis seed maturation. Plant Cell 18, 1642-1651. doi: 10.1105/tpc.105.039925

Tsuwamoto, R., Yokoi, S., and Takahata, Y. (2010). Arabidopsis EMBRYOMAKER encoding an AP2 domain transcription factor plays a key role in developmental change from vegetative to embryonic phase. Plant Mol. Biol. 73, 481-492. doi: 10.1007/s11103-010-9634-3

Varshney, R. K., Ribaut, J.-M., Buckler, E. S., Tuberosa, R., Rafalski, J. A., and Langridge, P. (2012). Can genomics boost productivity of orphan crops? Nat. Biotechnol. 30, 1172-1176. doi: 10.1038/nbt.2440

Viotti, C., Krüger, F., Krebs, M., Neubert, C., Fink, F., Lupanga, U., et al. (2013). The endoplasmic reticulum is the main membrane source for biogenesis of the lytic vacuole in Arabidopsis. Plant Cell 25, 3434-3449. doi: 10.1105/tpc.113.114827

Vitale, A., and Hinz, G. (2005). Sorting of proteins to storage vacuoles: how many mechanisms? Trends Plant Sci. 10, 316-323. doi: 10.1016/j.tplants.2005.05.001

Vitale, A., and Raikhel, N. V. (1999). What do proteins need to reach different vacuoles? Trends Plant Sci. 4, 149-155. doi: 10.1016/S1360-1385(99)01389-8

Voelker, C., Schmidt, D., Mueller-Roeber, B., and Czempinski, K. (2006). Members of the Arabidopsis AtTPK/KCO family form homomeric vacuolar channels in planta. Plant J. 48, 296-306. doi: 10.1111/j.1365-313X.2006.02868.x

Wang, J., Tse, Y. C., Hinz, G., Robinson, D. G., and Jiang, L. (2012). Storage globulins pass through the Golgi apparatus and multivesicular bodies in the absence of dense vesicle formation during early stages of cotyledon development in mung bean. J. Exp. Bot. 63, 1367-1380. doi: 10.1093/jxb/err366

Wang, X., Niu, Q.-W., Teng, C., Li, C., Mu, J., Chua, N.-H., et al. (2009). Overexpression of PGA37/MYB118 and MYB115 promotes vegetative-to-embryonic transition in Arabidopsis. Cell Res. 19, 224-235. doi: 10.1038/cr.2008.276

Wang, Y., Yu, B., Zhao, J., Guo, J., Li, Y., Han, S., et al. (2013). Autophagy contributes to leaf starch degradation. Plant Cell 25, 1383-1399. doi: 10.1105/tpc.112.108993

Willmann, M. R., Mehalick, A. J., Packer, R. L., and Jenik, P. D. (2011). MicroRNAs regulate the timing of embryo maturation in Arabidopsis. Plant Physiol. 155, 1871-1884. doi: 10.1104/pp.110.171355

Xiang, D., Venglat, P., Tibiche, C., Yang, H., Risseeuw, E., Cao, Y., et al. (2011). Genome-wide analysis reveals gene expression and metabolic network dynamics during embryo development in Arabidopsis. Plant Physiol. 156, 346-356. doi: 10.1104/pp.110.171702
Yang, H., Knapp, J., Koirala, P., Rajagopal, D., Peer, W. A., Silbart, L. K., et al. (2007). Enhanced phosphorus nutrition in monocots and dicots over-expressing a phosphorus-responsive type I H+-pyrophosphatase. Plant Biotechnol. J. 5, 735745. doi: 10.1111/j.1467-7652.2007.00281.x

Yano, K., Hattori, M., and Moriyasu, Y. (2007). A novel type of autophagy occurs together with vacuole genesis in miniprotoplasts prepared from tobacco culture cells. Autophagy 3, 215-221.

Zhang, H., and Ogas, J. (2009). An epigenetic perspective on developmental regulation of seed genes. Mol. Plant 2, 610-627. doi: 10.1093/mp/ssp027

Zhang, H., Wang, L., Deroles, S., Bennett, R., and Davies, K. (2006). New insight into the structures and formation of anthocyanic vacuolar inclusions in flower petals. BMC Plant Biol. 6:29. doi: 10.1186/1471-2229-6-29

Zhao, J., and Dixon, R. A. (2010). The 'ins' and 'outs' of flavonoid transport. Trends Plant Sci. 15, 72-80. doi: 10.1016/j.tplants.2009.11.006

Zheng, H., and Staehelin, L. A. (2011). Protein storage vacuoles are transformed into lytic vacuoles in root meristematic cells of germinating seedlings by multiple, cell type-specific mechanisms. Plant Physiol. 155, 2023-2035. doi: 10.1104/pp.110.170159

Zouhar, J., and Rojo, E. (2009). Plant vacuoles: where did they come from and where are they heading? Curr. Opin. Plant Biol. 12, 677-684. doi: 10.1016/j.pbi.2009.08.004

Zuo, J., Niu, Q.-W., Frugis, G., and Chua, N.-H. (2002). The WUSCHEL gene promotes vegetative-to-embryonic transition in Arabidopsis. Plant J. 30, 349-359. doi: 10.1046/j.1365-313X.2002.01289.x

Conflict of Interest Statement: The authors declare that the research was conducted in the absence of any commercial or financial relationships that could be construed as a potential conflict of interest.

Received: 28 September 2013; paper pending published: 21 October 2013; accepted: 15 November 2013; published online: 03 December 2013.

Citation: Feeney M, Frigerio L, Kohalmi SE, Cui Y and Menassa R (2013) Reprogramming cells to study vacuolar development. Front. Plant Sci. 4:493. doi: $10.3389 / \mathrm{fpls} .2013 .00493$

This article was submitted to Plant Cell Biology, a section of the journal Frontiers in Plant Science.

Copyright (c) 2013 Feeney, Frigerio, Kohalmi, Cui and Menassa. This is an openaccess article distributed under the terms of the Creative Commons Attribution License (CC BY). The use, distribution or reproduction in other forums is permitted, provided the original author(s) or licensor are credited and that the original publication in this journal is cited, in accordance with accepted academic practice. No use, distribution or reproduction is permitted which does not comply with these terms. 